\title{
Front Matter: Volume 9062
}

, "Front Matter: Volume 9062," Proc. SPIE 9062, Smart Sensor Phenomena, Technology, Networks, and Systems Integration 2014, 906201 (22 April 2014); doi: $10.1117 / 12.2064833$

Event: SPIE Smart Structures and Materials + Nondestructive Evaluation and Health Monitoring, 2014, San Diego, California, United States 


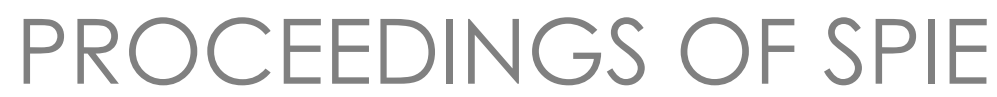

\title{
Smart Sensor Phenomena, Technology, Networks, and Systems Integration 2014
}

\author{
Wolfgang Ecke \\ Kara J. Peters \\ Norbert G. Meyendorf \\ Theodoros E. Matikas \\ Editors
}

10-11 March 2014

San Diego, California, United States

Sponsored by

SPIE

Cosponsored by

American Society of Mechanical Engineers (United States)

Cooperating Organizations

Intelligent Materials Forum (Japan)

Jet Propulsion Laboratory (United States)

National Science Foundation (United States)

Published by

SPIE 
The papers included in this volume were part of the technical conference cited on the cover and title page. Papers were selected and subject to review by the editors and conference program committee. Some conference presentations may not be available for publication. The papers published in these proceedings reflect the work and thoughts of the authors and are published herein as submitted. The publisher is not responsible for the validity of the information or for any outcomes resulting from reliance thereon.

Please use the following format to cite material from this book:

Author(s), "Title of Paper," in Smart Sensor Phenomena, Technology, Networks, and Systems Integration 2014, edited by Wolfgang Ecke, Kara J. Peters, Norbert G. Meyendorf, Theodoros E. Matikas, Proceedings of SPIE Vol. 9062 (SPIE, Bellingham, WA, 2014) Article CID Number.

ISSN: 0277-786X

ISBN: 9780819499882

Published by

SPIE

P.O. Box 10, Bellingham, Washington 98227-0010 USA

Telephone +1 3606763290 (Pacific Time) · Fax +1 3606471445

SPIE.org

Copyright (C) 2014, Society of Photo-Optical Instrumentation Engineers.

Copying of material in this book for internal or personal use, or for the internal or personal use of specific clients, beyond the fair use provisions granted by the U.S. Copyright Law is authorized by SPIE subject to payment of copying fees. The Transactional Reporting Service base fee for this volume is $\$ 18.00$ per article (or portion thereof), which should be paid directly to the Copyright Clearance Center (CCC), 222 Rosewood Drive, Danvers, MA 01923. Payment may also be made electronically through CCC Online at copyright.com. Other copying for republication, resale, advertising or promotion, or any form of systematic or multiple reproduction of any material in this book is prohibited except with permission in writing from the publisher. The CCC fee code is 0277-786X/14/\$18.00.

Printed in the United States of America.

Publication of record for individual papers is online in the SPIE Digital Library.

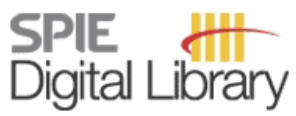

SPIEDigitalLibrary.org

Paper Numbering: Proceedings of SPIE follow an e-First publication model, with papers published first online and then in print and on CD-ROM. Papers are published as they are submitted and meet publication criteria. A unique, consistent, permanent citation identifier (CID) number is assigned to each article at the time of the first publication. Utilization of CIDs allows articles to be fully citable as soon as they are published online, and connects the same identifier to all online, print, and electronic versions of the publication. SPIE uses a six-digit CID article numbering system in which:

- The first four digits correspond to the SPIE volume number.

- The last two digits indicate publication order within the volume using a Base 36 numbering

system employing both numerals and letters. These two-number sets start with 00, 01, 02, 03, 04, $05,06,07,08,09,0 A, 0 B \ldots 0 Z$, followed by 10-1Z, 20-2Z, etc.

The CID Number appears on each page of the manuscript. The complete citation is used on the first page, and an abbreviated version on subsequent pages. Numbers in the index correspond to the last two digits of the six-digit CID Number. 


\section{Contents}

vii Conference Committee

\section{SESSION 1 DISTRIBUTED FIBER OPTIC SENSORS}

906202 OTDR and OFDR for distributed multi-parameter sensing (Invited Paper) [9062-1]

X. Bao, W. Li, Z. Qin, L. Chen, Univ. of Ottawa (Canada)

906203 Improved distributed fiber optic sensing system based on single-ended double-pulse input Brillouin scattering [9062-2]

T. Chang, R. Yang, Jilin Univ. (China); Y. Wang, D. Y. Li, L.C. Pegasus Corp. (China); L. Jia,

Shandong Univ. (China); H.-L. Cui, Jilin Univ. (China)

906204 Fiber optic temperature profiling for thermal protection heat shields [9062-40]

R. J. Black, J. M. Costa, B. Moslehi, L. Zarnescu, Intelligent Fiber Optic Systems Corp. (United States); D. Hackney, K. Peters, North Carolina State Univ. (United States)

906205 Vibration pattern recognition and classification in OTDR based distributed optical-fiber vibration sensing system [9062-4]

H. Zhu, C. Pan, X. Sun, Southeast Univ. (China)

\section{SESSION 2 FIBER OPTIC SENSORS}

906206 Localized measurements of composite dynamic response for health monitoring (Invited Paper) [9062-5]

S. Webb, K. Oman, K. Peters, North Carolina State Univ. (United States); N. Stan,

S. Chadderdon, R. Selfridge, S. Schultz, Brigham Young Univ. (United States)

906207 Piezo-optical measurements for guided wave and acoustic emission structural health monitoring [9062-6]

E. Frankforter, B. Lin, V. Giurgiutiu, Univ. of South Carolina (United States)

906208 Development of a wireless, self-sustaining damage detection sensor system based on chemiluminescence for structural health monitoring [9062-7]

K. S. C. Kuang, National Univ. of Singapore (Singapore)

$906209 \quad$ Novel optical fiber ultrasonic sensor based on fiber laser [9062-8]

Q. Wu, Y. Okabe, The Univ. of Tokyo (Japan); J. Sun, Huazhong Univ. of Science and

Technology (China)

\section{SESSION 3 MICROSTRUCTURED OPTICAL SENSORS}

9062 0A Fluorescence monitoring with steering wheel photonic crystal fiber (Invited Paper) [9062-9]

A. Mansaray, R. Wynne, Villanova Univ. (United States) 
9062 OB Image-based spectroscopy for environmental monitoring [9062-10]

E. Bachmakov, C. Molina, R. Wynne, Villanova Univ. (United States)

$90620 \mathrm{C}$ A novel microbending hetero-core fiber optic sensor for force and location sensing with applications to home security [9062-11]

S. Likoglu, K. Alemdar, K. Fidanboylu, O. Toker, Fatih Univ. (Turkey)

9062 OD A novel periodic macrobending hetero-core fiber optic sensor embedded in textile for respiratory movements' analysis [9062-12]

K. Alemdar, S. Likoglu, K. Fidanboylu, O. Toker, Fatih Univ. (Turkey)

9062 OE Millimeter-wave interferometry: an attractive technique for fast and accurate sensing of civil and mechanical structures [9062-14]

S. Kim, Information and Communications Univ. (Korea, Republic of); C. Nguyen, Texas A\&M Univ. (United States)

\section{SESSION 4 THERMOGRAPHY SENSORS}

9062 OF Pulsed thermographic inspection of CFRP structures: experimental results and image analysis tools [9062-15]

P. Theodorakeas, National Technical Univ. of Athens (Greece); N. P. Avdelidis, National Technical Univ. of Athens (Greece) and Univ. Laval (Canada); C. Ibarra-Castanedo, Univ. Laval (Canada); M. Koui, National Technical Univ. of Athens (Greece); X. Maldague, Univ. Laval (Canada)

9062 OG Thermo-electrical lockin thermography for characterization of subsurface defects [9062-16] E. Z. Kordatos, Sheffield Hallam Univ. (United Kingdom) and Univ. of Ioannina (Greece);

D. A. Exarchos, K. G. Dassios, T. E. Matikas, Univ. of loannina (Greece)

\section{SESSION 5 ELECTRIC FIELD SENSORS}

$9062 \mathrm{OH} \quad$ Non-intrusive electric field sensing (Invited Paper) [9062-17]

S. M. Schultz, R. Selfridge, S. Chadderdon, D. Perry, N. Stan, Brigham Young Univ. (United States)

9062 Ol Ion trap electric field measurements using slab coupled optical sensors [9062-18] L. Shumway, S. Chadderdon, A. Powell, A. Li, D. Austin, A. Hawkins, R. Selfridge, S. Schultz, Brigham Young Univ. (United States)

9062 0J Non-intrusive high voltage measurement using slab coupled optical sensors [9062-19] N. Stan, S. Chadderdon, R. H. Selfridge, S. M. Schultz, Brigham Young Univ. (United States)

9062 OK An ultrahigh sensitive self-powered current sensor utilizing a piezoelectric connected-inseries approach [9062-20]

P.-C. Yeh, T.-K. Chung, C.-H. Lai, National Chiao Tung Univ. (Taiwan) 
$9062 \mathrm{OL}$ Structural health monitoring on turbine engines using microwave blade tip clearance sensors (Invited Paper) [9062-21]

M. Woike, A. Abdul-Aziz, M. Clem, NASA Glenn Research Ctr. (United States)

$90620 \mathrm{M}$ Turbine engine rotor health monitoring evaluation by means of finite element analyses and spin tests data [9062-22]

A. Abdul-Aziz, M. R. Woike, M. Clem, G. Y. Baaklini, NASA Glenn Research Ctr. (United States)

$90620 \mathrm{~N}$ Progress of a cross-correlation based optical strain measurement technique for detecting radial growth on a rotating disk [9062-23]

M. M. Clem, M. R. Woike, NASA Glenn Research Ctr. (United States); A. Abdul-Aziz,

Cleveland State Univ. (United States)

906200 In-process, non-destructive multimodal dynamic testing of high-speed composite rotors [9062-24]

R. Kuschmierz, A. Filippatos, A. Langkamp, W. Hufenbach, J. W. Czarske, A. Fischer,

Technische Univ. Dresden (Germany)

\section{SESSION 7 ACOUSTIC EMISSION AND ULTRASOUND SENSORS}

9062 OP Acoustic emission signatures of damage modes in concrete [9062-25]

D. G. Aggelis, Vrije Univ. Brussel (Belgium); A. C. Mpalaskas, T. E. Matikas, Univ. of loannina (Greece); D. Van Hemelrijck, Vrije Univ. Brussel (Belgium)

$90620 Q \quad$ A qualitative and quantitative investigation of the uncracked and cracked condition of concrete beams using impulse excitation, acoustic emission, and ultrasonic pulse velocity techniques [9062-26]

S. lliopoulos, A. Iliopoulos, L. Pyl, H. Sol, D. G. Aggelis, Vrije Univ. Brussel (Belgium)

9062 OR Development of optical equipment for ultrasonic guided wave structural health monitoring [9062-27]

B. Lin, V. Giurgiutiu, Univ. of South Carolina (United States)

9062 OS Sensing light and sound velocities with phoxonic crystals [9062-28]

S. Amoudache, IEMN, CNRS, Univ. des Sciences et Technologies de Lille (France) and Univ. Mouloud Mammeri (Algeria); R. Moiseyenko, Y. Pennec, B. Djafari Rouhani, IEMN, CNRS, Univ. des Sciences et Technologies de Lille (France); A. Khater, Univ. du Maine (France); R. Lucklum, Otto-von-Guericke-Univ. (Germany); R. Tigrine, Univ. Mouloud Mammeri (Algeria)

9062 OT Measurement of elastic wave dispersion on human femur tissue [9062-29]

M. Strantza, Vrije Univ. Brussel (Belgium); O. Louis, Univ. Ziekenhuis Brussel, Vrije Univ. Brussel

(Belgium); D. Polyzos, Univ. of Patras (Greece); F. Boulpaep, D. Van Hemelrijck,

D. G. Aggelis, Vrije Univ. Brussel (Belgium) 
9062 OU Ultrasonic guided wave sensing properties of PVDF thin film with inter digital electrodes [9062-30]

V. T. Rathod, D. Roy Mahapatra, Indian Institute of Science (India)

POSTER SESSION

9062 OW 3D mapping of reinforcement and tendon ducts on pre-stressed concrete bridges by means of Ground Penetrating Radar (GPR) [9062-33]

E. Cheilakou, P. Theodorakeas, M. Koui, C. Zeris, National Technical Univ. of Athens (Greece)

$90620 \mathrm{X}$ Strength and fatigue life evaluation of composite laminate with embedded sensors [9062-34]

V. T. Rathod, S. R. Hiremath, D. Roy Mahapatra, Indian Institute of Science (India)

9062 OY A new type of microphone using flexoelectric barium strontium titnate [9062-35]

S. Kwon, W. Huang, North Carolina State Univ. (United States); S. Zhang, The Pennsylvania State Univ. (United States); F.-G. Yuan, X. Jiang, North Carolina State Univ. (United States)

$906202 \quad$ A new molecular dynamic model of nanowire motor [9062-36]

L. Wang, L. Li, H. Xu, G. Zhang, Q. Sun, Harbin Institute of Technology (China)

906210 The damage assessment methodology in cooperation with smart sensors and inspection robots [9062-37]

Y. Nitta, M. Ishida, T. Onai, Ashikaga Institute of Technology (Japan); M. Watakabe, Toda Corp. (Japan); A. Nishitani, C. Matsui, Waseda Univ. (Japan)

906212 Thermal and electrical behavior of nano-modified cement mortar [9062-39]

D. A. Exarchos, P. T. Dalla, I. K. Tragazikis, P. Alafogianni, N.-M. Barkoula, A. S. Paipetis,

K. G. Dassios, T. E. Matikas, Univ. of Ioannina (Greece)

906213 Fiber-optically sensorized composite wing [9062-41]

J. M. Costa, R. J. Black, B. Moslehi, L. Oblea, R. Patel, V. Sotoudeh, Intelligent Fiber Optic Systems Corp. (United States); E. Abouzeida, V. Quinones, Y. Gowayed, P. Soobramaney, G. Flowers, Auburn Univ. (United States)

906214 Lamb wave-based damage detection of composite shells using high-speed fiber-optic sensing [9062-42]

V. Sotoudeh, R. J. Black, B. Moslehi, Intelligent Fiber Optic Systems Corp. (United States);

P. Qiao, Washington State Univ. (United States)

906215 Fast fiber Bragg grating interrogation system with scalability to support monitoring of large structures in harsh environments [9062-43]

B. Moslehi, R. J. Black, J. M. Costa, E. H. Edwards, F. Faridian, V. Sotoudeh, Intelligent Fiber Optic Systems Corp. (United States)

906216 Thermal diffusivity estimation of templated nanocomposite using frequency modulated infrared imaging [9062-44]

L. Indu Giri, S. Tuli, Indian Institute of Technology Delhi (India)

Author Index

vi 


\section{Conference Committee}

Symposium Chairs

Victor Giurgiutiu, University of South Carolina (United States)

Christopher S. Lynch, University of California, Los Angeles

(United States)

Symposium Co-chairs

Jayanth N. Kudva, NextGen Aeronautics, Inc. (United States)

Theodoros E. Matikas, University of loannina (Greece)

Conference Chair

Wolfgang Ecke, Institut für Photonische Technologien e.V. (Germany)

Conference Co-Chairs

Kara J. Peters, North Carolina State University (United States)

Norbert G. Meyendorf, Fraunhofer IKTS-CMD (Germany) and University of Dayton (United States)

Theodoros E. Matikas, University of loannina (Greece)

Conference Program Committee

Farhad Ansari, University of Illinois at Chicago (United States)

George Y. Baaklini, NASA Glenn Research Center (United States)

Horst J. Baier, Technische Universität München (Germany)

Curtis E. Banks, NASA Marshall Space Flight Center (United States)

Xiaoyi Bao, University of Ottawa (Canada)

Hartmut Bartelt, Institut für Photonische Technologien e.V. (Germany)

Rolf Brönnimann, EMPA (Switzerland)

Brian Culshaw, University of Strathclyde (United Kingdom)

Chiara Daraio, ETH Zurich (Switzerland)

Wolfgang R. Habel, Bundesanstalt für Materialforschung und-prüfung (Germany)

Daniele Inaudi, Smartec S.A. (Switzerland)

Kerop D. Janoyan, Clarkson University (United States)

YeonWan Koh, FIBERPRO, Inc. (Korea, Republic of)

Silvio E. Kruger, National Research Council Canada (Canada)

Jinsong Leng, Harbin Institute of Technology (China)

Alexis Mendez, MCH Engineering LLC (United States)

Bernd Michel, Fraunhofer-Institut für Elektronische Nanosysteme

(Germany) 
Jeff W. Miller, Micron Optics, Inc. (United States)

Marc Nikles, Omnisens S.A. (Switzerland)

loannis E. Psarobas, University of Athens (Greece)

Richard Selfridge, Brigham Young University (United States)

Leszek Roman Jaroszewicz, Military University of Technology (Poland)

Nobuo Takeda, The University of Tokyo (Japan)

Michael D. Todd, University of California, San Diego (United States)

Eric Udd, Columbia Gorge Research (United States)

Zhishen Wu, Ibaraki University (Japan)

Rosalind M. Wynne, Villanova University (United States)

Chung-Bang Yun, Ulsan National Institute of Science and Technology (Korea, Republic of)

\section{Session Chairs}

1 Distributed Fiber Optic Sensors

Wolfgang Ecke, Institut für Photonische Technologien e.V. (Germany)

2 Fiber Optic Sensors

Kara J. Peters, North Carolina State University (United States)

Wolfgang Ecke, Institut für Photonische Technologien e.V. (Germany

3 Microstructured Optical Sensors

Kara J. Peters, North Carolina State University (United States)

4 Thermography Sensors

Theodoros E. Matikas, University of loannina (Greece)

5 Electric Field Sensors

Stephen Schultz, Brigham Young University (United States)

6 Sensors for SHM of Turbine Engines

Mark R. Woike, NASA Glenn Research Center (United States)

7 Acoustic Emission and Ultrasound Sensors

Norbert G. Meyendorf, Fraunhofer IKTS-CMD (Germany) and University of Dayton (United States) 